\title{
Iron-loss Calculation of Inductors Considering Non-uniform Flux Density Conditions
}

\author{
Hiroaki Sato* Member, Yoshihiro Miwa* Student Member \\ Toshihisa Shimizu*a) $^{*}$ Fellow, Kinji Kanagawa** Member
}

(Manuscript received July 13, 2018, revised Oct. 2, 2018)

\begin{abstract}
Owing to the significant advances made in the field of power semiconductor devices, such as $\mathrm{SiC}$ and $\mathrm{GaN}$, the switching frequency of power converters has been increased. Thus, the volume of passive components such as filter inductors and transformers can be reduced. However, the reduction in the heat-dissipation surface area of inductors results in a considerable increase in temperature, which is a constraint for increasing the power density of power converters. To prevent these problems, the iron-loss evaluation of inductors is one of the most important issues in realizing high-power-density converters. This paper presents an accurate iron-loss calculation method for inductors considering the non-uniformity of the flux-density distribution in the magnetic core. First, it is verified that the non-uniformity of the flux-density distribution in the core results in the deviation of the iron-loss measurement even when the same magnetic material is used in the core and when the inductor is excited under the same excitation condition. Next, the procedure to obtain the iron-loss data, which describes the relationship between the iron loss and excitation condition more precisely, is introduced. Finally, a method to accurately calculate the iron loss in an inductor using electromagnetic simulation and more precise iron-loss data is introduced. method is verified using a toroidal core and a UU core under sine voltage condition and rectangular voltage condition.
\end{abstract}

Keywords: iron loss, DC pre-magnetization, inductor, air gap, electromagnetic simulation

\section{Introduction}

Recently, there have been several advances in the field of power semiconductor devices, such as $\mathrm{SiC}$ and $\mathrm{GaN}$, and the switching frequency of power converters has been increased. As a result, the volume of the magnetic components, such as filter inductors and transformers, can be reduced. However, a reduction of the heat-dissipation surface area of magnetic components results in an increase in the temperature, and this causes an increase in the power density of the power converters. To overcome this problem, the accurate evaluation of the iron loss in inductors is one of the most important issues to be addressed when realizing high-power-density converters ${ }^{(1)-(4)}$.

There have been many studies on iron-loss calculations for transformers and motors ${ }^{(5)-(8)}$, and accurate iron-loss calculations have been performed. However, there are nonnegligible calculation errors in the iron-loss calculation for the inductors used in the power converters. This is because the conventional loss-calculation methods neglect the influence of dc pre-magnetization on the iron loss. To overcome this issue, the authors have previously proposed a lossmap method ${ }^{(9)-(13)}$, which is an iron-loss calculation method

a) Correspondence to: Toshihisa Shimizu. E-mail: shimizut@tmu. ac.jp

* Tokyo Metropolitan University

1-1, Minami-Osawa, Hachioji, Tokyo 192-0397, Japan

${ }^{* *}$ Mitsubishi Materials Corporation

1-600, Kitabukuro-cho, Omiya-ku, Saitama-shi, Saitama 3308508, Japan for inductors using a loss map. The loss map describes the measured iron-loss characteristic curve for excitation conditions such as dc pre-magnetization. One of the parameters in the loss map is dc pre-magnetization, and thus the iron loss can be calculated considering the influence of the dc pre-magnetization. With the loss-map method, the iron loss in inductors that are used in a buck converter or in a pulsewidth modulation (PWM) inverter can be calculated accurately ${ }^{(9)-(13)}$.

However, the loss-map method neglects the influence on the iron loss of the non-uniformity of the flux-density distribution in the core. It is generally known that the value of the flux density in the core is different for each section in the core. Nevertheless, to simplify the calculation, the following two assumptions are used in the loss-map method.

A) When measuring the iron loss in an inductor under measurement to derive the loss map, the flux-density distribution in the core is assumed to be uniform.

B) When calculating the iron loss in an inductor, under measurement, the flux-density distribution in the core used in the inductor is assumed to be uniform.

Assumption A) leads to a deviation in the iron-loss values on the loss map resulting from ignoring the non-uniformity of the flux-density distribution in the core. This means that the conventional loss map does not describe precisely the correspondence between the iron loss and excitation condition. In addition, assumption B) leads to a calculation error when determining the iron loss in an inductor where the flux-density distribution is complex, such as a gapped inductor. 
To overcome these problems, this paper first presents the procedure to derive a loss map that describes more precisely the relationship between iron losses and the excitation condition (hereafter, such a loss map is referred to as a more precise loss map). In addition, this paper also proposes a method to calculate the iron loss in inductors considering the non- uniformity of the flux-density distribution in the core. This method is performed by using the more precise loss map and electromagnetic simulation in the core. This paper is organized as follows. The loss-map method and the iron-loss measurement system are briefly introduced in Section 2. In Section 3, the experiment and electromagnetic simulations results are presented to verify that the flux-density distribution in the core affects the iron-loss measurement. In Section 4 , the adjusting coefficient, which makes the conversion from the conventional loss map to the more precise loss map, is proposed. Section 5 introduces an approach to calculate the iron loss in inductors that use both the more precise loss map and electromagnetic simulation in the core, and the method is experimentally verified. Finally, Section 6 concludes this paper.

\section{Loss-Map Method}

2.1 Iron Loss in Inductors Figures 1 and 2 show the general buck-converter circuit and the operation waveform of the inductor, respectively. As shown in Fig. 2, the inductor current $i_{L}$ includes a dc component $I_{L, d c}$; the magnetic field $H$ includes a dc pre-magnetization $H_{d c}$. From the results, the hysteresis curve is positioned in the first quadrant of the $\mathrm{B}-\mathrm{H}$ plane, as shown in Fig. 3. This curve is referred to as a minor loop, and the area corresponds to the iron loss per unit volume, $Q\left[\mathrm{~J} / \mathrm{m}^{3}\right]$. The area of a minor loop varies not only with the flux-density ripple, $\Delta B$, and the switching frequency, $f_{s w}$, but also with $H_{d c}$. In other words, the iron loss in inductors varies with $\Delta B, f_{s w}$, and $H_{d c}$. Figure 4 shows the iron-loss characteristic curve as a function of $H_{d c}$ for the ferrite (PC40 TDK). In this paper, this iron-loss characteristic curve is referred to as a loss map. The iron-loss data in the loss map is measured using the iron loss measurement system discussed in Subsection 2-2. As shown in Fig. 4, the iron loss varies nonlinearly with $H_{d c}$, and the characteristic for $H_{d c}$ depends largely on magnetic materials ${ }^{(14)}$. Therefore, the influence of $H_{d c}$ on the iron loss need to be considered when calculating the iron loss in inductors.

2.2 Iron Loss Measurement System Figure 5 shows the system that is employed to measure iron losses under rectangular voltage and dc pre-magnetized condition. This system is also used to derive the loss map. This system consists of a B-H analyzer (SY-8219 IWATSU) and an external dc voltage source. The B-H analyzer excites the inductor under measurement with a rectangular voltage. The external dc voltage source injects a desired dc current to the inductor current. As a result, the inductor under measurement is excited with a triangular current including the dc component, as shown in Fig. 2. Then, the B-H analyzer calculates automatically the iron loss $P[\mathrm{~W}]$ using the following equation.

$$
P=\frac{1}{T_{s}} \int_{0}^{T_{s}} i_{L} v_{L} d t \ldots \ldots \ldots \ldots \ldots \ldots \ldots \ldots \ldots
$$

where $T_{s}$ is the switching cycle, $i_{L}$ is the inductor current, and

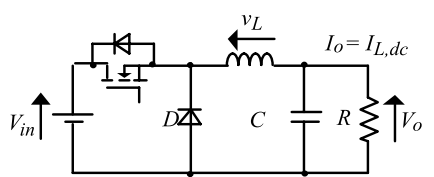

Fig. 1. General buck converter circuit

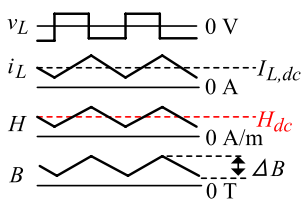

Fig. 2. Operation waveform of the inductor used in a general buck converter

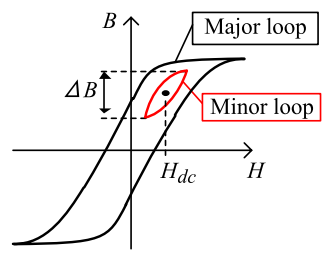

Fig. 3. Major and minor loops

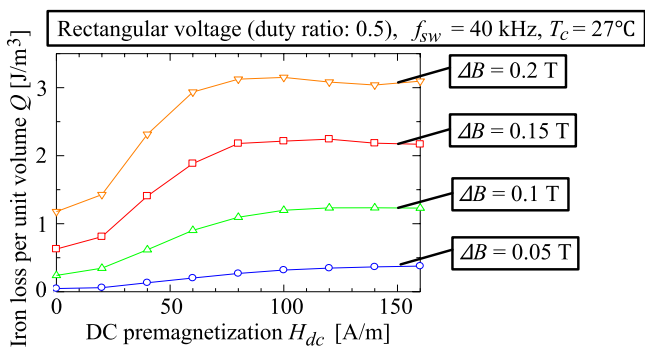

Fig. 4. Loss map for ferrite core (PC40 TDK)

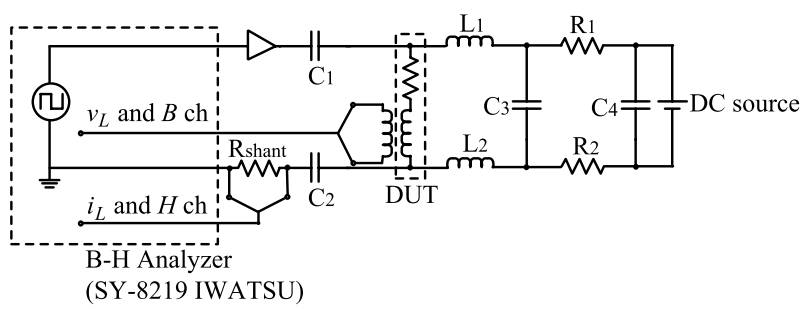

Fig. 5. Iron-loss measurement system under rectangular voltage and dc pre-magnetized condition

Table 1. Specifications of the iron-loss measurement system

\begin{tabular}{|l|c|}
\hline Ripple inductor current & $6 \mathrm{~A}$ \\
\hline DC bias current & $30 \mathrm{~A}$ \\
\hline Inductor voltage & $\pm 200 \mathrm{~V}$ \\
\hline Switching frequency & $10 \mathrm{kHz}-1 \mathrm{MHz}$ \\
\hline
\end{tabular}

$v_{L}$ is the inductor voltage. The specifications of this measurement system are shown in Table 1 . To measure only the iron loss with this system, the two-winding method is used. $v_{L}$ is measured with the secondary winding, which has a high impedance. Thus, the measured iron loss does not include the copper loss.

To derive the loss map, the iron losses should be measured under various excitation conditions, i.e., various values of $\Delta B, H_{d c}$, and $f_{s w}$. In the B-H analyzer, $\Delta B$ and $H_{d c}$ are defined as in the following equations under the assumption that 
the flux density $B$ and $H$ are uniform in the core.

$$
\begin{aligned}
\Delta B & =\frac{1}{N A_{c}} \int_{0}^{d T_{s}} v_{L} d t=\frac{d V_{L, p}}{N A_{c} f_{s w}} \cdots \ldots \ldots \ldots \ldots \ldots \ldots \ldots \ldots \ldots \ldots \ldots \ldots \ldots \ldots \ldots \ldots
\end{aligned}
$$

where $d$ is the duty ratio, $V_{L, p}$ is the peak value of the primary inductor voltage, $A_{c}$ is the cross-sectional area of the core, $f_{s w}$ is the switching frequency, $I_{L, d c}$ is the dc component of the inductor current, and $l_{c}$ is the flux-path length of the core. (2) and (3) are based on Faraday's law and Ampère's law, respectively. As shown in (2) and (3), the iron loss can be measured under desired $\Delta B, H_{d c}$, and $f_{s w}$. by adjusting $V_{L, p}, I_{L, d c}$, and $f_{\text {sw. }}$.

When measuring the iron loss in the inductor, the phase error between the inductor current measurement and the inductor voltage measurement causes a significant decrease in the measurement accuracy. In this system, the phase error is corrected automatically by the $\mathrm{B}-\mathrm{H}$ analyzer with respect to each frequency component contained in the captured waveform. The principle is discussed in detail in (1). The phase error after correction is \pm 0.15 degree within the frequency range $10 \mathrm{kHz}-10 \mathrm{MHz}$.

The iron loss varies with the magnetic material temperature. Therefore, the temperature of the inductor being measured should be kept constant. In this system, one measurement takes approximately $10 \mathrm{~s}$. In addition, sufficient cooling time is provided between measurements. Therefore, the temperature of the inductor is always kept at room temperature, a condition under which all of the iron-loss data in this study were measured.

2.3 Loss-map Method The derived loss map is used in the loss-map method. The loss-map method is a method that calculates the iron loss in the inductor considering the influence of the dc pre-magnetization on the iron loss. As an example, this paper introduces the procedure to calculate the iron loss in an inductor used in the buck converter, as shown in Fig. 6. In a previous step, the circuit condition and the specification of the inductor under design is determined. In addition, the loss map, which corresponds to the magnetic

Previous step

- Chose the circuit condition and the specification of the inductor under design.

- Obtain the loss map by measuring the iron loss in the inductor fabricated with a general toroidal core.

Step 1

Calculate the excitation condition, such as the dc premagnetization $H_{d c}$ and flux-density ripple $\Delta B$ using (2) and (3), respectively.

\begin{tabular}{l} 
Step 2 \\
Obtain the iron loss per unit volume $Q_{V}\left[\mathrm{~J} / \mathrm{m}^{3}\right]$ corresponding \\
to the excitation condition from the loss map. \\
$\begin{array}{l}\text { Step } 3 \\
\text { Calculate the whole iron loss } P[\mathrm{~W}] \text { using (4). }\end{array}$ \\
\hline
\end{tabular}

Fig. 6. Procedure for the conventional loss-map method material used as the core in the inductor under design, must be measured with a simple-shape core, e.g., toroidal core. Next, the excitation condition, such as $\Delta B$ and $H_{d c}$, are calculated using (2) and (3), respectively. Then, it is assumed that the distribution of the flux density and magnetic field in the core is uniform. After that, the iron loss per unit volume, $Q_{V}\left[\mathrm{~J} / \mathrm{m}^{3}\right]$, which corresponds to the excitation condition, is obtained from the loss map. Finally, the whole iron loss $P[\mathrm{~W}]$ is calculated using the following equation.

$$
P=Q_{V} V_{c} f_{s w} \ldots \ldots \ldots \ldots \ldots \ldots \ldots \ldots \ldots \ldots \ldots \ldots \ldots \ldots
$$

where $V_{c}$ is the volume of the core.

As discussed in subsections 2-2 and 2-3, (2) and (3) are respectively used to determine $\Delta B$ and $H_{d c}$ in the loss-map method. However, these equations are only true under the assumption that the flux-density distribution in the core is uniform. As a result, the conventional loss map is expected to have deviations that are caused by not considering the nonuniformity of the flux-density distribution in the core. Moreover, when calculating the iron loss in the inductor where the flux-density distribution is complex, calculation errors are expected.

\section{Influence of Non-uniformity of Flux-density Distribution on Iron-loss Measurement}

In this section, experimental verifications are first presented to show that the iron-loss measurement is affected by the difference in the specification of the core used in the inductor under measurement, even under the same excitation condition. Next, it is shown that this caused by ignoring the non-uniformity of the flux-density distribution in the core by applying the electromagnetic simulation to the core.

3.1 Experimental Verification To verify that the non-uniformity of the flux-density distribution affects the iron-loss measurement and the loss map, two cores shown in Figs. 7(a) and (b) were used. Test core 1, which is shown in Fig. 7(a), is a general toroidal core; on the other hand, test core 2, which is shown in Fig. 7(b), was specially fabricated for this experiment. The specification of both cores is shown in Table 2. Both cores are made by an iron powder (MBS-R3 Mitsubishi Materials), and the only difference between them is the diameter ratio $n$, which is defined as

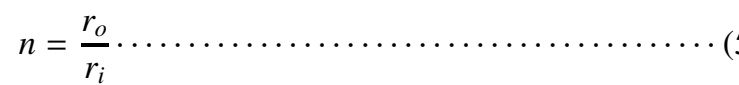

where $r_{i}$ is the inside radius of the core and $r_{o}$ is the outer radius of the core. The value $n$ of test core 1 shown in Fig. 7(a) is higher than that of test core 2, which is shown in Fig. 7(b). This means that the difference between the magnetic flux at

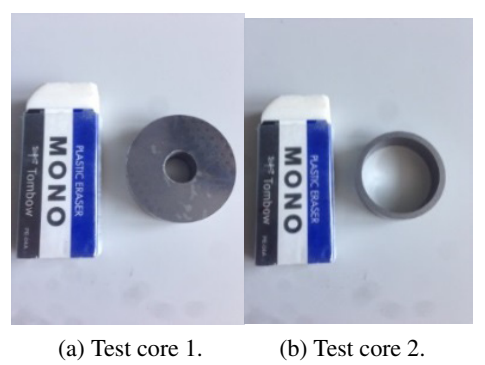

Fig. 7. Exterior view of test core 
Table 2. Specification of the cores shown in Fig. 7

\begin{tabular}{|c|c|c|}
\hline & Core 1 & Core 2 \\
\hline Inside radius $r_{i}[\mathrm{~mm}]$ & 5.0 & 13.0 \\
\hline Outer radius $r_{o}[\mathrm{~mm}]$ & 17.5 & 15.0 \\
\hline Thickness $t[\mathrm{~mm}]$ & 10.0 & 10.0 \\
\hline Diameter ration $n$ & 3.5 & 1.15 \\
\hline Core material & \multicolumn{2}{|c|}{ Iron dust (MBS-R3 } \\
& \multicolumn{2}{|c|}{ Mitsubishi materials) } \\
\hline
\end{tabular}

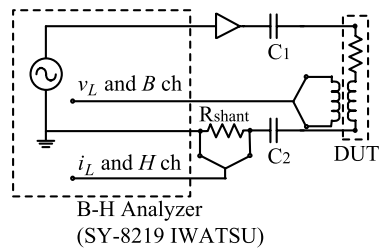

Fig. 8. Iron-loss measurement system under sine voltage

Table 3. Specification of the iron-loss measurement system shown in Fig. 8

\begin{tabular}{|l|c|}
\hline Ripple inductor current & $6 \mathrm{~A}$ \\
\hline DC bias current & $30 \mathrm{~A}$ \\
\hline Inductor voltage & $\pm 200 \mathrm{~V}$ \\
\hline Switching frequency & $10 \mathrm{kHz}-1 \mathrm{MHz}$ \\
\hline
\end{tabular}

Table 4. Measurement conditions

\begin{tabular}{|c|c|}
\hline Voltage waveform & Sin \\
\hline The peak value of the flux density $B_{p}[\mathrm{mT}]$ & $1 \sim 350$ \\
\hline Switching frequency $f_{s w}[\mathrm{kHz}]$ & 10 \\
\hline Temperature of the core $T_{c}\left[{ }^{\circ} \mathrm{C}\right]$ & 27 \\
\hline
\end{tabular}

the inner side of the core, which is where the flux density is strongest, and that at the outer side of the core, where the flux density is a minimum, is larger in test core 1 than in test core 2. Therefore, the influence of ignoring the non-uniformity of the flux-density distribution is more significant in test core 1 than in test core 2 . The iron loss per unit volume $P_{V}\left[\mathrm{~W} / \mathrm{m}^{3}\right]$ in these test cores was measured and compared under the sine voltage condition.

The iron-loss measurement system shown in Fig. 8 was used to measure the iron loss under the sine voltage condition. The specification of the measurement system is shown in Table 3. The fundamental principles, such as the second winding method and the procedure to handle the phase error, are the same as the iron-loss measurement system shown in Fig. 5. In this system, the peak value of the flux density $B_{p}$ is defined as follows under the assumption that the flux-density distribution in the core is uniform.

$$
B_{p}=\frac{1}{N A_{c}} \int_{0}^{\frac{T_{s}}{4}} v_{L} d t \ldots \ldots \ldots \ldots \ldots \ldots \ldots \ldots \ldots
$$

where $N$ is the primary winding number, $A_{c}$ is the crosssectional area of the core, $T_{s}$ is the switching cycle, and $v_{L}$ is the inductor voltage. By adjusting $v_{L}$, the iron loss under the desired $B_{p}$ condition can be measured.

Table 4 shows the measurement condition, and Fig. 9 shows the measurement results of $P_{V}\left[\mathrm{~W} / \mathrm{m}^{3}\right]$ in test core 1 and test core 2. As shown in Fig. $9, P_{V}$ values measured with test core 1 are lower than those measured with test core

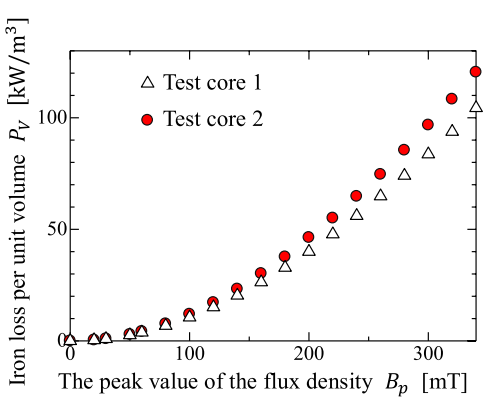

Fig. 9. Iron-loss characteristic curve as a function of $B_{p}$ of test core

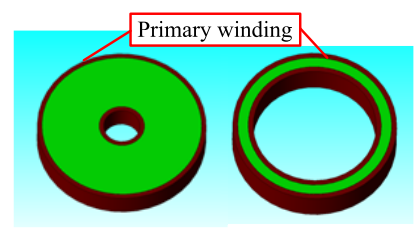

$\begin{array}{ll}\text { (a) Test core } 1 & \text { (b) Test core } 2\end{array}$

Fig. 10. Simulation models for test cores shown in Fig. 7 with winding

Table 5. Specifications of the simulation models shown in Fig. 10

\begin{tabular}{|c|c|c|}
\hline & Core 1 & Core 2 \\
\hline Inside radius $r_{i}[\mathrm{~mm}]$ & 5.0 & 13.0 \\
\hline Outer radius $r_{o}[\mathrm{~mm}]$ & 17.5 & 15.0 \\
\hline Thickness $t[\mathrm{~mm}]$ & 10.0 & 10.0 \\
\hline Diameter ration $n$ & 3.5 & 1.15 \\
\hline Core material & Iron dust (MBS-R3 Mitsubishi Materials) \\
\hline Mesh number & 2303 & 21418 \\
\hline
\end{tabular}

2. This result verifies that the difference in the specification of the core used in the inductor under measurement results in the deviation in the iron-loss measurement and loss map, even under the same excitation condition.

3.2 Electromagnetic Simulation in the Core To better understand the reason for the deviation, the flux-density distributions in test core 1 and test core 2 were simulated using the finite-element method (FEM). JMAG JSOL was used for the electromagnetic simulation. Figures 10(a) and (b) show the simulation model with winding for test core 1 and test core 2, respectively. The specifications of both simulation models are shown in Table 5, and the other values, except for the mesh number, are the same as that of Table 2. The mesh numbers are chosen considering both the simulation time and the simulation accuracy. The winding is modeled as a single-turn copper sheet with thickness $t_{w}=1 \mathrm{~mm}$ in simulation models in order to avoid the deviation of the flux density resulting from the non-uniformity of the winding arrangement. In order to avoid making the simulation time unnecessary long, the simulation does not consider the eddy current in the conductor or the diamagnetic field due to the eddy current.

To compare the flux-density distribution in these simulation models under the same flux-density condition as in the experiment, $B_{p}$ is determined by using the following equation in the simulation

$$
B_{p}=\frac{\phi_{p}}{A_{c}}
$$




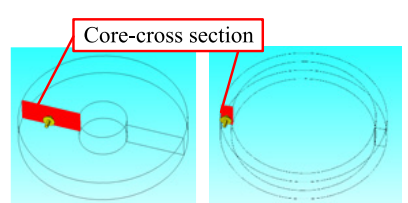

$\begin{array}{ll}\text { (a) Test core } 1 & \text { (b) Test core } 2\end{array}$

Fig. 11. Core- cross section used to define the flux density in the core

Table 6. Simulation conditions

\begin{tabular}{|c|c|}
\hline Voltage waveform & Sin \\
\hline Peak value of the flux density $B_{p}[\mathrm{mT}]$ & 50 \\
\hline Switching frequency $f_{s w}[\mathrm{kHz}]$ & 10 \\
\hline Temperature of the core $T_{c}\left[{ }^{\circ} \mathrm{C}\right]$ & 27 \\
\hline
\end{tabular}

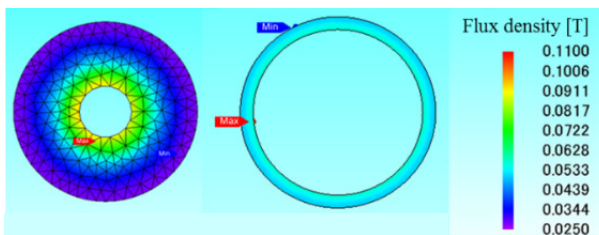

(a) Test core 1

(b) Test core 2

Fig. 12. Simulation results of the magnetic flux-density distribution in test core

where $\phi_{p}$ is the peak value of the whole flux that interlinks the cross section, which is shown in Fig. 11, in the simulation model. The cross-sectional area of the core $A_{c}$ in (7) corresponds to the cross-sectional area of the simulation model for the core. $\phi_{p}$ can be adjusted to be the same value as the experiment by adjusting the peak value of the inductor current $I_{L, p}$ that flows through the winding on simulation.

Table 6 shows the simulation condition, and Figs. 12(a) and (b) show the simulation results of the flux-density distribution in the core. As shown in Fig. 12(b), the flux-density distribution in test core 2 is almost uniform. However, that of test core 1 is non-uniform. This results show that the iron loss per unit volume $P_{V}$ of test core 1 is smaller than that of test core 2 because the volume of the outer part of test core 1 , where a lower iron loss is generated, is relatively large.

In conclusion, it was verified that the non-uniformity of the flux-density distribution affects the iron-loss measurement and loss map. Moreover, the iron losses that are measured with a core where the diameter ratio is small, such as test core 2 , describes more precisely the correspondence between the iron loss and the excitation condition because the flux density is almost the same in such a core. Therefore, the more precise loss map can be derived by measuring the iron loss in a core where the diameter ratio is small.

\section{Adjusting Coefficient to Derive a More Precise Loss Map}

As discussed in Section 3, a core with a small diameter ratio $n$ is necessary to derive the more precise loss map. However, such a special core cannot always be obtained. Therefore, this section proposes the adjusting coefficient that makes the conversion from the loss map derived with a common toroidal core to the more precise loss map.

4.1 Derivation of Adjusting Coefficient To derive the adjusting coefficient, the two core models shown in

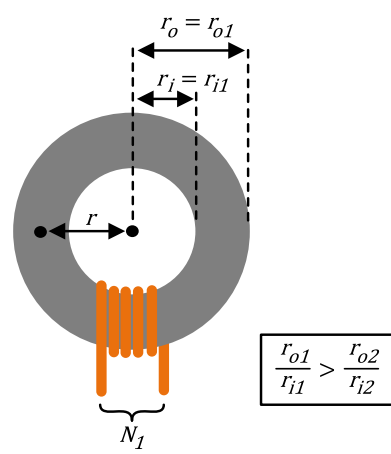

(a) Core model 1

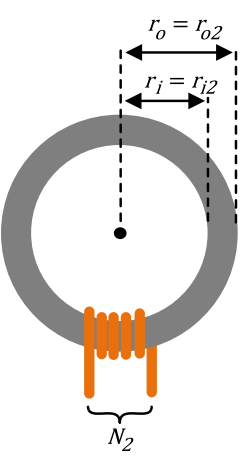

(b) Core model 2 .
Fig. 13. Core model with winding used to derive adjusting coefficient

Fig. 13 were used. The flux-path length $l_{c}$, the thickness $t$, and the magnetic material are assumed to be the same in both models. The only difference between the two models is the diameter ratio $n$, which is defined as (5). The diameter ratio of core model $1, n_{1}$, is higher than that of core model 2 , $n_{2}$. By using the iron loss per unit volume of core model 1 $P_{V 1}\left[\mathrm{~W} / \mathrm{m}^{3}\right]$ and that of core model $2 P_{V 2}\left[\mathrm{~W} / \mathrm{m}^{3}\right]$, the adjusting coefficient is defined as follows:

$$
m=\frac{P_{V 2}}{P_{V 1}} \ldots \ldots \ldots \ldots \ldots \ldots \ldots \ldots \ldots \ldots \ldots \ldots \ldots \ldots
$$

$P_{V 2}$, which describes precisely the correspondence between the iron loss and the excitation condition, is obtained by multiplying $m$ by the $P_{V 1}$ value measured using a general toroidal core, such as core model 1. $P_{V 1}$ and $P_{V 2}$ must be first described as an equation to derive the adjusting coefficient $m$. Next, the procedure to describe $P_{V 1}$ and $P_{V 2}$ in equation form is introduced.

When core 1 is excited under the sine voltage condition, $P_{V 1}$ is determined as

$$
P_{V 1}=\frac{P_{1}}{V_{c 1}}=\frac{1}{V_{c 1}} \int_{0}^{t_{1}} \int_{r_{i 1}}^{r_{o 1}} P_{V} d V_{c} \ldots \ldots \ldots \ldots \ldots
$$

where $P_{1}$ is the whole iron loss of core model $1, V_{c 1}, t_{1}, r_{i 1}$ and $r_{o 1}$ are the volume, the thickness, the inside radius and the outer radius of core model 1 , respectively. The iron loss in the microcore volume $P_{V} d V_{c}[\mathrm{~W}]$ is determined as in the following equation according to the Steinmetz equation ${ }^{(15)}$

$$
P_{V} d V_{c}=k f_{s w}^{\alpha} B_{r, p}^{\beta} d V_{c}
$$

where $k, \alpha$, and $\beta$ are the Steinmetz parameters, which depend on the magnetic material, $f_{s w}$ is the switching frequency and $B_{r, p}$ is the peak value of the flux density at a distance from the center of the core $r\left(r_{i 1} \leq r \leq r_{o 1}\right)$. $B_{r, p}$ is determined as in the following equation according to Ampère's law

$$
B_{r, p}=\frac{\mu_{r} \mu_{o} N_{1} I_{L, p}}{2 \pi r} \ldots \ldots \ldots \ldots \ldots \ldots \ldots \ldots \ldots \ldots \ldots
$$

where $\mu_{r}$ is the relative permeability of the core, $\mu_{o}$ is the space permeability, $N_{1}$ is the winding number wound to core model 1 , and $I_{L, p}$ is the peak value of the inductor current. According to (15), the value of $\beta$ depends on the magnetic material. However, in typical magnetic materials, the value of $\beta$ is approximately 2 . Under the assumption that $\beta=2$, (9) 
is described as in the following equation by substituting (10) and (11) for (9).

$$
P_{V 1}=\frac{k f_{s w}{ }^{\alpha} t_{1}\left(\mu_{r} \mu_{o} N_{1} I_{L, p}\right)^{2} \ln n_{1}}{2 \pi V_{c 1}}
$$

By performing a similar procedure, $P_{V 2}$ is described as

$$
P_{V 2}=\frac{k f_{s w}{ }^{\alpha} t_{2}\left(\mu_{r} \mu_{o} N_{2} I_{L, p}\right)^{2} \ln n_{2}}{2 \pi V_{c 2}}
$$

where $t_{2}$ is the thickness of the core model 2, $N_{2}$ is the winding number wound to core model 2 , and $V_{c 2}$ is the volume of core model 2. When comparing (12) and (13), the relationship between the ampere turn $N_{1} I_{L, p}$ and $N_{2} I_{L, p}$ is not clear. Therefore, it is difficult to compare $P_{V 1}$ and $P_{V 2}$ under the same excitation condition. To solve this problem, the peak value of the flux density $B_{p}$ defined as (7) is used. By using (11), (7) is described as in the following equation for core model 1

$$
B_{p}=\frac{t_{1}}{t_{1}\left(r_{o 1}-r_{i 1}\right)} \int_{r_{i 1}}^{r_{o 1}} B_{r, p} d r=\frac{\mu_{r} \mu_{o} N_{1} I_{L, p} \ln n_{1}}{2 \pi\left(r_{o 1}-r_{i 1}\right)}
$$

By eliminating $\mu_{r}, \mu_{o}, N_{p 1}$, and $I_{L, p}$ from (12) and (14), $P_{V 1}$ is given as

$$
P_{V 1}=\frac{2\left(n_{1}-1\right)}{\ln n_{1}\left(n_{1}+1\right)} k f_{s w}{ }^{\alpha} B_{p}^{2} .
$$

By performing a similar procedure, $P_{V 2}$ is described as

$$
P_{V 2}=\frac{2\left(n_{2}-1\right)}{\ln n_{2}\left(n_{2}+1\right)} k f_{s w}{ }^{\alpha} B_{p}{ }^{2} .
$$

When the excitation condition, such as $f_{s w}$ and $B_{p}$, are the same, $m$ is described as in the following equation by eliminating $k, f_{s w}{ }^{\alpha}$ and $B_{p}{ }^{2}$ from (15) and (16).

$$
m=\frac{\left(n_{2}-1\right)\left(n_{1}+1\right) \ln n_{1}}{\left(n_{2}+1\right)\left(n_{1}-1\right) \ln n_{2}} .
$$

4.2 Experimental Verification By using $m$, the iron loss per unit volume $P_{V}\left[\mathrm{~W} / \mathrm{m}^{3}\right]$ measured with test core 1 , as shown in Fig. 7(a), was converted to that of test core 2, as shown in Fig. 7(b). These iron loss data have already been presented in Fig. 9. The conversion result is shown in Fig. 14. As shown in Fig. 14, the iron loss data measured with test core 2 and the converted result are in good agreement. The error between both results is believed to be caused by the

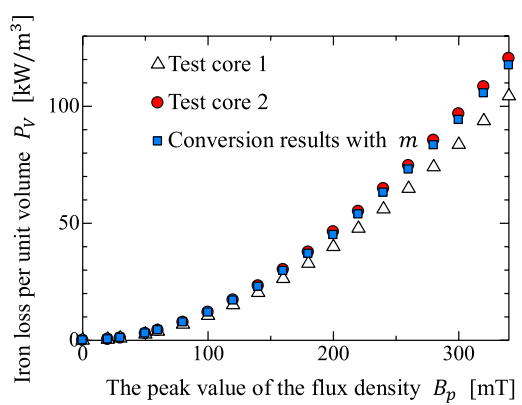

Fig. 14. Conversion result of iron loss per unit volume using the adjusting coefficient $m$ individual difference between cores. From this result, the effectiveness of the conversion coefficient $m$ was verified. The advantage of using $m$ is that the precise iron loss data can be obtained without the need for a specially fabricated core, such as test core 2 .

As discussed above, the adjusting coefficient $m$ is derived only under the sine voltage condition. The adjusting coefficient under the dc pre-magnetized condition should be also derived; however, it is difficult. When deriving the adjusting coefficient $m$, the effectin (11) must be solved. Nevertheless, the equation of $P_{V 1}$ for $H_{d c}$, which is introduced in (2), is too complex to solve the integral in (11). The method to derive the adjusting coefficient corresponding to $H_{d c}$ will be the focus of future work.

\section{Iron-loss Calculation Method Considering the Non-uniformity of the Flux-density Distribu- tion}

This section introduces the method to calculate the iron loss in inductors considering the non-uniformity of the fluxdensity distribution.

5.1 Procedure for the Proposed Method Figure 15 shows the procedure that is followed for the proposed method. The following explains the difference between the proposed method and the conventional loss-map method shown in Fig. 6, which is improved in order to calculate the iron loss in inductors considering the non-uniformity of the flux-density distribution.

A) In the previous step, a more precise loss map was derived using a core where the diameter ratio is small.

B) In steps 1-3, the excitation condition and the iron loss are calculated with respect to each micro volume in the core using electromagnetic simulations.

In step 1 in Fig. 15, the simulation model for the inductor under design is first designed. Next, the magnetic field $H$ and

Previous step

- Chose the circuit condition and the specification of the inductor under design.

- Obtain a more precious loss map by measuring the iron loss in the inductor fabricated with a toroidal core where the diameter ratio is small.

Step 1
Design the simulation model for the inductor under design, and
calculate the excitation condition with respect to each mesh part
in the simulation model by performing electromagnetic
simulations.

Step 2

Obtain the iron loss per unit volume $Q_{V}\left[\mathrm{~J} / \mathrm{m}^{3}\right]$ with respect to each mesh part corresponding to the excitation condition from the more precise loss map.

Step 3

Calculate the whole iron loss $P[\mathrm{~W}]$ by summing the iron losses for mesh part $P_{m e s h}[\mathrm{~W}]$.

Fig. 15. Procedure for calculating the iron loss in inductors considering the non-uniformity of the flux-density distribution 
Calculation of Iron Losses Considering Non-uniform Flux Density (Hiroaki Sato et al.)

Table 7. Experimental conditions

\begin{tabular}{|c|c|c|c|c|c|}
\hline Experiment number. & 1 & 2 & 3 & 4 & 5 \\
\hline Voltage waveform & Sine & Sine & Triangular & Triangular & Triangular \\
\hline Magnetic material & $\begin{array}{c}\text { Iron dust (MBS-R3 } \\
\text { Mitsubishi Materials) }\end{array}$ & $\begin{array}{c}\text { Iron dust (MBS-R3 } \\
\text { Mitsubishi Materials) }\end{array}$ & $\begin{array}{c}\text { Iron dust (MBS-R3 } \\
\text { Mitsubishi Materials) }\end{array}$ & $\begin{array}{c}\text { Iron dust (MBS-R3 } \\
\text { Mitsubishi Materials) }\end{array}$ & Ferrite (PC40 TDK) \\
\hline Core shape & Toroidal & UU & Toroidal & UU & Toroidal \\
\hline Air gap length $l_{g}[\mathrm{~mm}]$ & 0 & $0.3,0.5,1.0$ & 0 & $0.3,0.5,1.0$ & 0.9 \\
\hline Switching frequency $f_{s w}[\mathrm{kHz}]$ & 10 & 10 & 10 & 10 & 40 \\
\hline Peak value of the flux density $B_{p}[\mathrm{mT}]$ & 50,100 & 50 & Not defined & Not defined & Not defined \\
\hline Flux-density ripple $\Delta B[\mathrm{mT}]$ & Not defined & Not defined & 100,200 & 100 & 111 \\
\hline $\begin{array}{c}\text { DC pre-magnetization } H_{d c}[\mathrm{~A} / \mathrm{m}] \text { or } \\
\text { DC component of inductor current } \\
I_{L, d c}[\mathrm{~A}]\end{array}$ & 0 & 0 & $H_{d c}=300 \mathrm{~A} / \mathrm{m}$ & $I_{L, d c}=0.8 \mathrm{~A}$ & $I_{L, d c}=6 \mathrm{~A}$ \\
\hline
\end{tabular}

the flux density $B$ are simulated with respect to each mesh under a desired condition using electromagnetic simulations. Then, the flux-density ripple for mesh, $\Delta B_{\text {mesh }}$, and the dc pre-magnetization for mesh $H_{m e s h, d c}$ are respectively defined as in the following equations.

$$
\begin{aligned}
& \Delta B_{\text {mesh }}=B_{\text {mesh,max }}-B_{\text {mesh }, \text { min }} \cdots \\
& H_{\text {mesh }, d c}=\frac{H_{\text {mesh }, \text { max }}+H_{m e s h, \text { min }}}{2}
\end{aligned}
$$

where $B_{\text {mesh,max }}$ and $B_{\text {mesh,min }}$ are respectively the maximum and minimum values of the flux density under steady-state conditions. In addition, $H_{\text {mesh,max }}$ and $H_{\text {mesh,min }}$ are respectively the maximum and minimum values of the magnetic field under steady-state conditions. $B_{\text {mesh,max }}, B_{\text {mesh,min }}$, $H_{\text {mesh,max }}$ and $H_{\text {mesh,min }}$ are automatically calculated with respect to each mesh by performing electromagnetic simulations. In step 2, the iron loss per unit volume, $Q_{V}\left[\mathrm{~J} / \mathrm{m}^{3}\right]$, which corresponds to $\Delta B_{m e s h}$ and $H_{m e s h, d c}$, is obtained from the more precise loss map under the condition of $\Delta B=$ $\Delta B_{m e s h}$ and $H_{d c}=H_{m e s h, d c}$. This determination of $Q_{V}$ is conducted with respect to each mesh. In step 3, the iron loss for mesh $P_{\text {mesh }}$ is first calculated as in the following equation with respect to each mesh.

$$
P_{\text {mesh }}=Q_{V} V_{\text {mesh }} f_{s w}
$$

where $V_{\text {mesh }}$ is the mesh volume and $f_{s w}$ is the switching frequency. Finally, the whole iron loss $P$ that is generated in the core is calculated as

$$
P=\sum P_{m e s h}
$$

As discussed in subsection 5-2, the experimental verification was first conducted under a simple excitation condition, i.e., the sine voltage condition. In this case, the peak value of the flux density for mesh $B_{\text {mesh, }}$ was simulated instead of $\Delta B_{\text {mesh }}$ and $H_{\text {mesh }, d c}$ in step 1. Moreover, in step 2, a more precise loss map corresponding to the sine voltage condition was used in order to determine $Q_{V}$.

5.2 Experimental Verification To verify the suitability of the proposed method, experiments were conducted under various conditions. Table 7 shows all of the experimental conditions. Experiments 1 and 2 were conducted under the sine voltage condition, and experiments 3, 4 and 5 were performed under rectangular voltage and dc pre-magnetized conditions. In experiments $1-4$, iron powder core (MBS-R3
Mitsubishi Materials) was used in the inductor under measurement. In addition, an air gap was inserted into the core in experiments 2 and 4 to verify the usefulness of the proposed method under complex flux-density distributions. Moreover, the ferrite core (PC40 TDK), where the iron loss is sensitive for dc pre-magnetization as shown in Fig. 4, is used in experiment 5. The excitation conditions shown in Table 7, such as $f_{s w}, B_{p}, \Delta B$, and $H_{d c}$, were determined based on the general operating range of the power electronics application using $\mathrm{Si}$ and/or $\mathrm{SiC}$ as well as the properties of the magnetic material.

JMAG JSOL was used as the electromagnetic simulation software. In addition, the iron-loss measurement system shown in Fig. 8 and Fig. 5 were used to measure iron loss under the sine voltage condition as well as under rectangular voltage and dc pre-magnetized conditions, respectively. The calculation and experimental results should be compared under the same $B-H$ condition and the same $i_{L^{-}} v_{L}$ condition. However, in the electromagnetic simulation, it is difficult to obtain the exact same $B-H$ condition as that of the experiment even when the simulation model is simulated under the same $i_{L}-v_{L}$ condition as that of the experiment. Moreover, this paper focuses on the usefulness of the more precise loss map and the iron-loss calculation with respect to each tiny volume. Therefore, in this paper, the calculation and simulation results were compared under the same $B_{p}$, and $\Delta B$ condition instead of the same $i_{L}-v_{L}$ condition. When comparing the results under the sine voltage condition, $B_{p}$ in the experiment and in the simulation are defined as (6) and (7), respectively. The adjustment of $B_{p}$ to be the same value in the simulation and experiment has been discussed in Subsection 3.2. When comparing the results under the rectangular voltage and dc pre-magnetized condition, $\Delta B$ is defined as (2) in the experiment, respectively, while $\Delta B$ in the simulations are respectively defined as in the following equations.

$$
\Delta B=\frac{\phi_{\max }-\phi_{\min }}{A_{c}} \cdots \ldots \ldots \ldots \ldots \ldots \ldots \ldots \ldots \ldots \ldots \ldots \ldots \ldots
$$

where $\phi_{\max }$ and $\phi_{\min }$ are the maximum and minimum values under steady-state conditions of the whole flux, which interlinks the cross section of the simulation model for core. $A_{c}$ is the cross-sectional area of the simulation model for core. In the simulation, the values of $\Delta B$ was adjusted to be the same as in the experiment by adjusting the ripple component of the inductor current that flows through the winding wound to the simulation model for core. Figure 16 illustrates the value of whole flux $\phi$ under steady state and the flux-density 


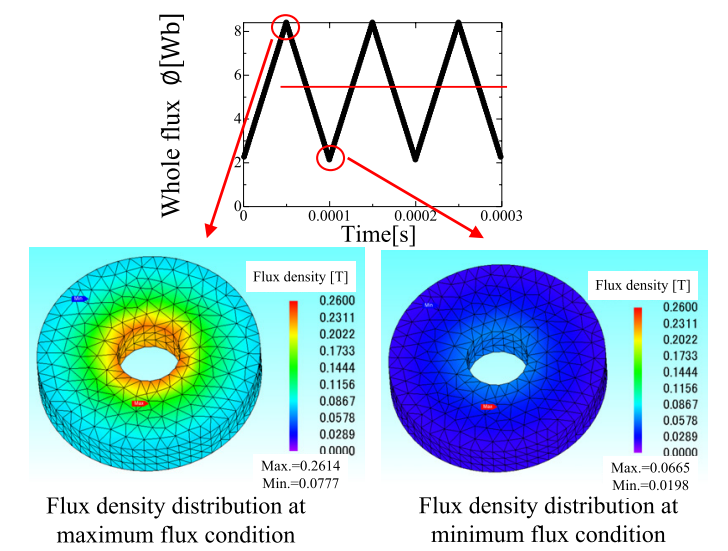

Fig. 16. Flux-density distribution under maximum and minimum magnetizing conditions

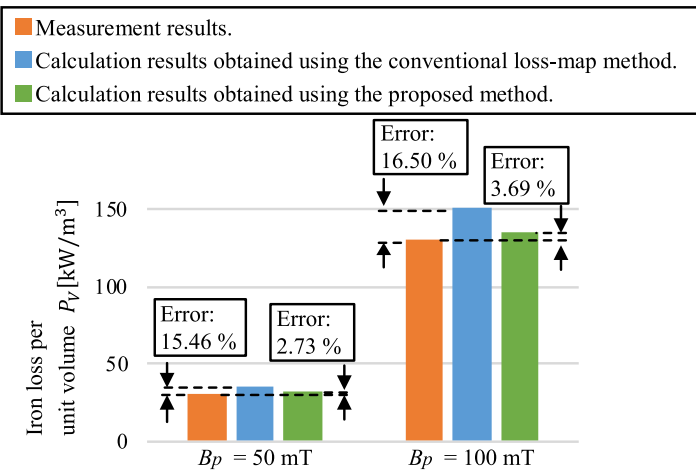

Fig. 17. Iron loss in the inductor fabricated with the general toroidal core shown in Fig. 7(a) under the sine voltage condition $\left(f_{s w}=10 \mathrm{kHz}\right)$

distribution when $\phi=\phi_{\max }$ and $\phi=\phi_{\min }$ in simulation. Additionally, the dc component of inductor current in simulation was adjusted to the same value as that of experiment in order to simulate dc pre-magnetized condition. The value of $H_{d c}$ shown in experiment 3 in Table 7 is calculated with (3). On the other hand, the experimental value of the dc component of inductor current is shown in Table 7 in the case of Experiment 4 and 5 This is because it is difficult to calculate accurately $H_{d c}$ with (3) when air gap is inserted to the core.

In experiment 1 , the iron loss in the general toroidal core shown in Fig. 7(a) is calculated and measured under the sine voltage condition. The more precise loss map corresponding to the magnetic material (MBS-R3) and sine voltage condition was derived by measuring the iron loss in the core shown in Fig. 7(b). Figure 17 shows the calculation and measurement results. The maximum error between the measurement result and the result calculated by the proposed method is $3.69 \%$. Moreover, the error decreases by $12.81 \%$ at the maximum compared with the value when using the conventional loss-map method shown in Fig. 6.

In experiment 2, the UU shaped core shown in Fig. 18 was used. The specification of the UU shaped core is shown in Table 8 . The calculation and experiment were conducted under the sine voltage condition and for various air-gap conditions (the air-gap length $l_{g}=0.3,0.5$, and $1.0 \mathrm{~mm}$ ). Each value of the air-gap length is the total value of two air gaps. Figure 19 shows the exterior view of the inductor where the UU shaped

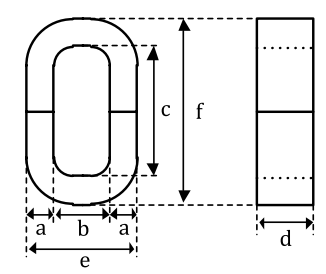

Fig. 18. UU-shaped core

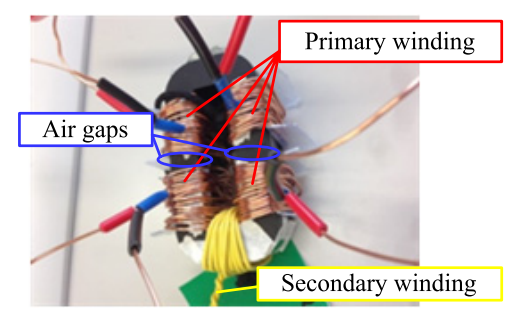

Fig. 19. Exterior view of the inductor where the UUshaped core is used

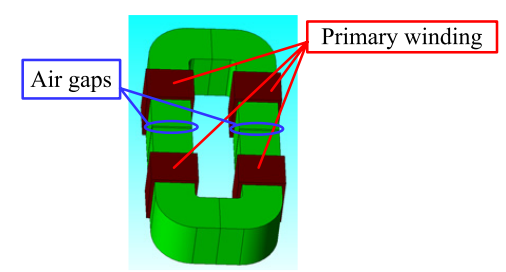

Fig. 20. Simulation model for the UU-shaped iron core and windings

core is used. The primary winding and secondary winding are wound to the inductor. The fringing loss caused by the fringing flux is one of the losses encountered in gapped inductors; however, in this study, the increase in iron losses resulting from the fringing loss was neglected for simplicity. Therefore, the experiment was conducted under low fringing loss conditions. The fringing losses can be classified as:

A) Losses in the core surface because of the eddy currents caused by the interlinkage of the fringing flux and core.

B) Losses in the winding because of the interlinkage of the fringing flux and winding

The fringing losses in A) can be neglected because both the iron dust and MnZn ferrite cores used in the experiment have a high electrical resistance. The fringing losses classified in B) can be prevented by separating the winding from the air gap; the experimental inductors have been designed in that way. Moreover, to preventing the winding arrangement from affecting the flux-density distribution in the core, the primary winding is wound to be as uniform as possible. Figure 20 shows the simulation model for the UU-shaped core with winding. The specification of the simulation model is the same as that of the real core shown in Table 8, and the number of meshes in the simulation model is 18785 . Figure 21 shows the simulation result of the flux density in the core when $B_{p}=50 \mathrm{mT}$ and $l_{g}=0.5 \mathrm{~mm}$. As shown in Fig. 21, the flux density on the inside of the corner is higher than that outside the corner. Moreover, the flux density decreases as the portion gets closer to the air-gap portion. This is because of the fringing flux. Figure 22 shows the calculation and experiment results of the iron loss in the core. As shown in Fig. 22, the error between calculation results and measurement results 
Table 8. Specifications of the UU-shaped core

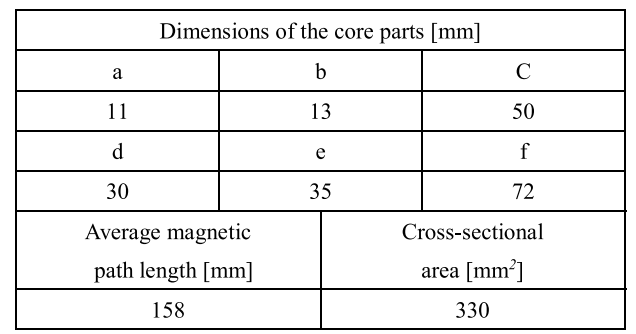

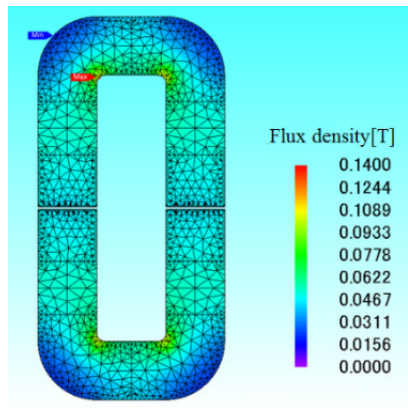

Fig. 21. Simulated result for the magnetic flux-density distribution in the UU-shaped core with an air gap $\left(f_{s w}=\right.$ $10 \mathrm{kHz}, B_{p}=50 \mathrm{mT}$ )

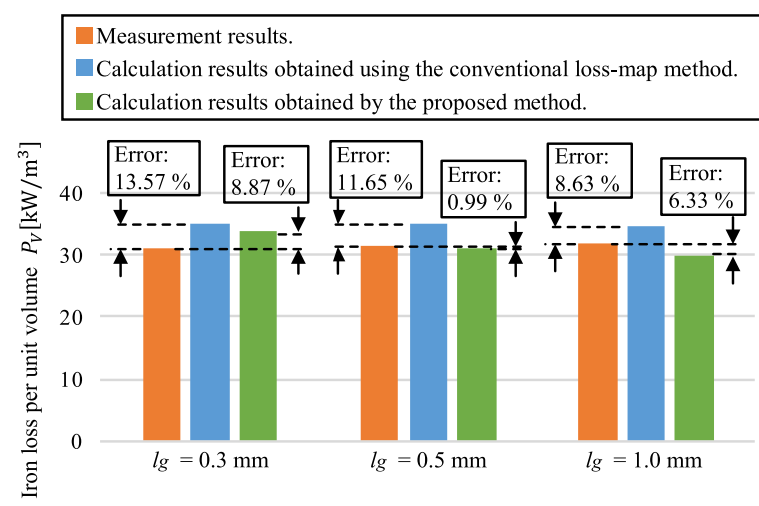

Fig. 22. Iron loss in the inductor shown in Fig. 18 under sine voltage condition $\left(f_{s w}=10 \mathrm{kHz}, B_{p}=50 \mathrm{mT}\right)$

decreases under all air-gap conditions by using the proposed method.

In experiments 3 and 4 , the calculation and experiment results of the iron loss are compared under rectangular voltage and dc pre-magnetized conditions. The core shapes are toroidal and UU. These cores and the inductors are the same as those used in experiment 1 and experiment 2 , respectively. The more precise loss map corresponding to the rectangular voltage and dc pre-magnetized condition is shown in Fig. 23. This loss map was obtained by measuring the iron loss of the test core 2 shown in Fig. 7(b). The conventional loss map derived with test core 1 is also shown in Fig. 23. The iron loss value of the more precise loss map is higher than that of the conventional loss map by about $10 \%$ as with Fig. 9 . Figure 24 shows the calculation and measurement results of the iron loss in the inductor where the toroidal core shown in Fig. 7(a) is used. The maximum error between the measurement result and the result calculated by the proposed method is $1.10 \%$. Moreover, the error decreases by $13.16 \%$ at the maximum compared to the case when using the conventional

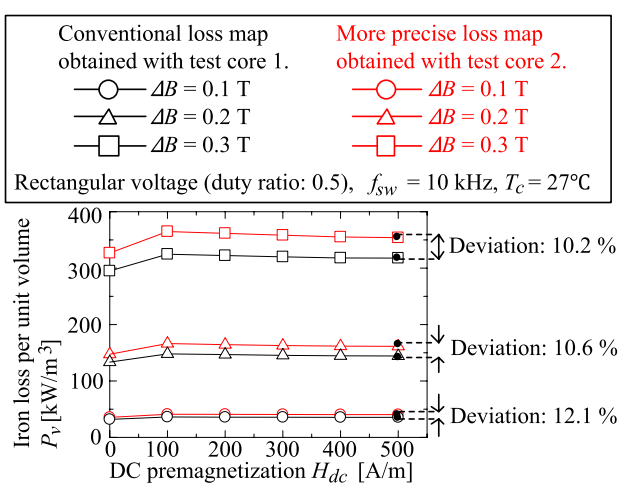

Fig. 23. More precise loss map and conventional loss map for iron dust (MBS-R3 Mitsubishi Materials)

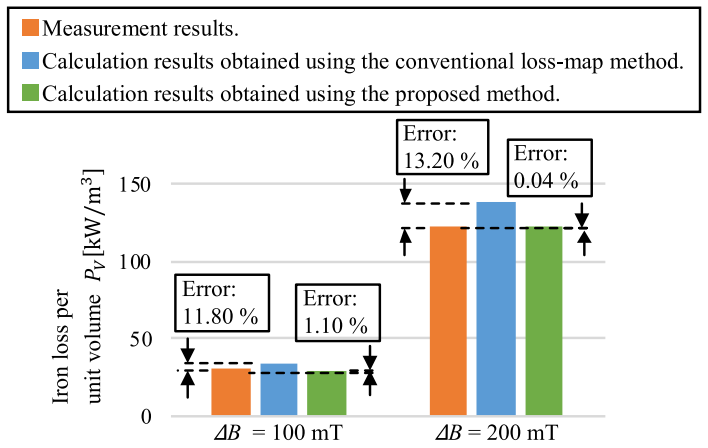

Fig. 24. Iron loss in the inductor fabricated with the general toroidal core shown in Fig. 7(a) under rectangular voltage and dc pre-magnetized condition $\left(f_{s w}=10 \mathrm{kHz}\right)$

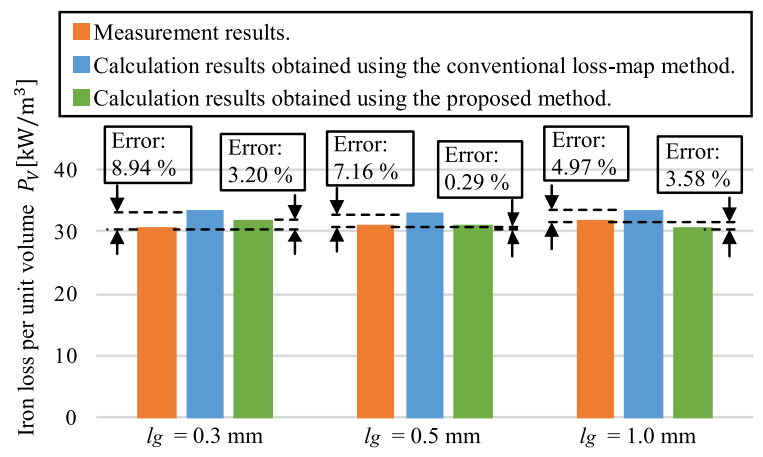

Fig. 25. Iron loss in the inductor shown in Fig. 18 under rectangular voltage and dc pre-magnetized condition $\left(f_{s w}=10 \mathrm{kHz}, \Delta B=50 \mathrm{mT}\right)$

loss-map method. Figure 25 shows the calculation and measurement results of the iron loss in the UU-shaped core. As shown in the figure, by using the proposed method, the error between the calculation results and measurement results decreases under all air-gap conditions.

Finally, the experimental verification for the ferrite core (PC40 TDK) was conducted under rectangular voltage and dc pre-magnetized conditions. Air gaps are often inserted into ferrite cores to prevent magnetic saturation. Therefore, in this study, an air gap $\left(l_{g}=0.9 \mathrm{~mm}\right)$ was inserted into the core. Figure 26 shows the exterior view of the inductor that is fabricated in the ferrite core. The specification of the core is shown in Table 9. The loss map used in the proposed method should be derived by measuring the iron loss in the core where the diameter ratio is small. However, ferrite cores 


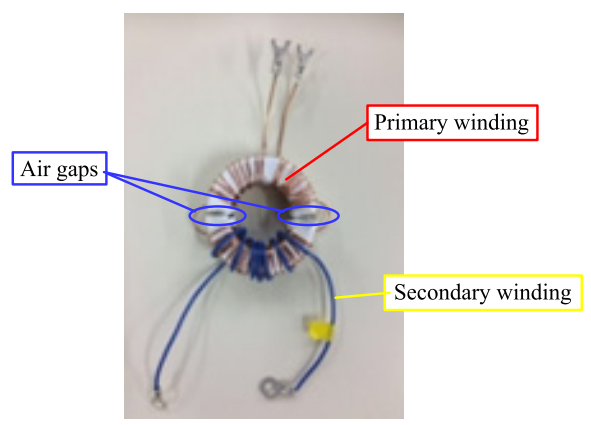

Fig. 26. Exterior view of the inductor fabricated with ferrite core (PC 40 TDK)

Table 9. Specifications of the inductor shown in Fig. 26

\begin{tabular}{|c|c|}
\hline Inside radius [mm] & 15.5 \\
\hline Outer radius [mm] & 25.5 \\
\hline Thickness [mm] & 13.0 \\
\hline Air gap [mm] & 0.9 \\
\hline Number of turns & 43 \\
\hline
\end{tabular}

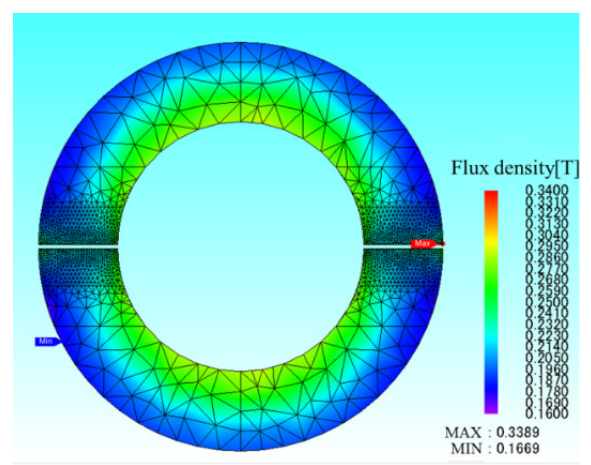

Fig. 27. Simulation result of the magnetic flux-density distribution in the core used in the inductor shown in Fig. $25\left(f_{s w}=40 \mathrm{kHz}\right)$

Table 10. Iron loss in the inductor shown in Fig. 26

\begin{tabular}{|c|c|}
\hline Calculated value $\left[\mathrm{kW} / \mathrm{m}^{3}\right]$ & 0.443 \\
\hline Measured value $\left[\mathrm{kW} / \mathrm{m}^{3}\right]$ & 0.437 \\
\hline Error & $1.37 \%$ \\
\hline
\end{tabular}

are not easily processed to such a core. Therefore, the loss map was derived by measuring the iron loss of a core where the diameter is smaller among commercially available. The core is same as the core used in the inductor shown in Fig. 26. The loss map measured by the core was already shown in Fig. 4. Figure 27 shows the simulation result of the magnetic flux-density distribution in the core used in the inductor shown in Fig. 26, which shows that the flux density on the inner side of the core is higher than that of the outer side of the core. Table 10 shows the measurement result and the result calculated by the proposed method. Both results are in good agreement, and the error between both results is $1.37 \%$.

From these results, the suitability of the proposed method is verified under both the sine voltage condition and rectangular voltage condition. Moreover, the iron loss in the inductors fabricated with the gapped core can be calculated using the proposed method.

\section{Conclusion}

In this paper, it is first shown that the non-uniformity of the flux-density distribution in the core affects iron-loss measurements. This means that the loss map that is derived based on iron-loss measurements contains deviations, and the ironloss calculation with the loss map causes errors that result from the deviation. To overcome these issues, the following are proposed in this paper:

A) The Adjusting coefficient to derive a more precise loss map.

B) An iron-loss calculation method considering the nonuniformity of the flux-density distribution in the core by using a more precise loss map and electromagnetic simulations.

Finally, the proposed method was verified under sine and rectangular voltage conditions. In particular, the iron loss in gapped cores, where the flux-density distribution is complex, can be calculated with high accuracy.

\section{References}

( 1 ) S. Iyasu, T. Shimizu, and K. Ishii: "A Novel Inductor Loss Calculation Method on Power Converters Based on Dynamic Minor Loop", IEEJ Trans. Ind. Appl., Vol.126, No.7, pp.1028-1034 (2006)

( 2 ) J. Muhlethaler, J. Biela, J. Kolar, and A. Ecklebe: "Core Losses Under the DC Bias Condition Based on Steinmetz Parameters", IEEE Trans. Power Electron., Vol.27, No.2, pp.953-963 (2012)

( 3 ) A. Krings and J. Soulard: "Overview and Comparison of Iron Loss Models for Electrical Machines", MC2D \& MITI EVER, Monaco (2011)

( 4 ) T. Maeda, et al.: "Development of Super Low Iron-loss P/M Soft Magnetic Material", SEI Technical Review - English Edition- 60 (2005)

( 5 ) N. Hyuk, et al.: "A Study on Iron Loss Analysis Method Considering the Harmonics of the Flux Density Waveform Using Iron Loss Curves Tested on Epstein Samples", Magnetics, IEEE Trans., Vol.39. No.3, pp.1472-1475 (2003)

( 6 ) M. Kawabe, et al.: "Behavior of Minor Loop and Iron Loss Under Constant Voltage Type PWM Inverter Excitation”, Magnetics, IEEE Trans., Vol.48, No.11, pp.3458-3461 (2012)

( 7 ) H. Kaihara, et al.: "Effect of Carrier Frequency and Circuit Resistance on Iron Loss of Electrical Steel Sheet Under Single-phase Full-bridge PWM Inverter Excitation", Magnetics, IEEE Trans., Vol.48, No.11, pp.3454-3457 (2012)

( 8 ) R. Kaczmarek, M. Amar, and F. Protat: "Iron Loss Under PWM Voltage Supply on Epstein Frame and in Induction Motor Core", Magnetics, IEEE Trans., Vol.32, No.1, pp.189-194 (1996)

( 9 ) S. Iyasu, T. Shimizu, and K. Ishii: "Iron Loss Calculation Method of Filter Inductor Core on a Single-Phase PWM Voltage Source Inverter", IEEJ Trans. Ind. Appl., Vol.127, No.3, pp.217-225 (2007)

(10) K. Kakazu, T. Shimizu, et al.: "Verification of Iron Loss Calculation Method using a High-Precision Iron Loss Analyzer", IEEJ Trans. Ind. Appl., Vol.133, No.1, pp.84-93 (2013)

(11) H. Matsumori, T. Shimizu, K. Takano, and H. Ishii: "Iron Loss Calculation of AC Filter Inductor for Three-phase PWM Inverters", IEEJ Trans. Ind. Appl., Vol.133, No.10, pp.1009-1021 (2013)

(12) T. Shimizu, K. Kakazu, T. Takano, and H. Ishii: "Verification of Iron Loss Calculation Method using a High-Precision Iron Loss Analyzer", IEEJ Trans. Ind. Appl., Vol.133, No.1, pp 84-93 (2013)

(13) Y. Miwa, T. Shimizu, K. Takano, and H. Ishii: "Study on Low-loss Design of Inductor Used for Buck-chopper Circuit Based on Iron Loss Characteristic Considering Pre-magnetization", IEEJ Trans. Ind. Appl., Vol.137, No.5, pp.385-394 (2017)

(14) H. Matsumori, T. Shimizu, K. Takano, and H. Ishii: "Iron Loss Evaluation of Filter Inductor for Non-sinusoidal and Pre-magnetization”, IEEJ IAS Conf., pp.I465-I468 (2015)

(15) C. Steinmetz: "On the Law of Hysteresis", Proc. of the IEEE, Vol.72, No.2, pp.197-221 (1984) 
Hiroaki Sato (Member) received the B.S. and M.S. degrees in elec-

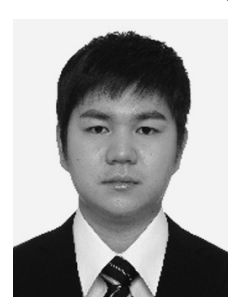
trical engineering from Tokyo Metropolitan University, Japan in 2014 and 2016, respectively. He is currently an engineer concerned with the design and development of automobiles in Honda Motor co., ltd. His research interests ware calculating the iron losses in magnetic components and the design of inductors used in power electronics circuits when he was a student in Tokyo Metropolitan University.

Yoshihiro Miwa

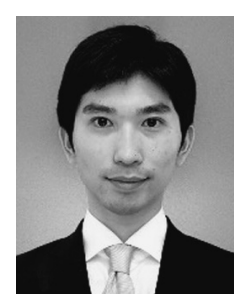

(Student Member) received the B.S. and M.S. degrees in electrical engineering from Tokyo Metropolitan University, Japan in 2014 and 2016, respectively, and is currently pursuing the Ph.D. degree at the same university. His research interests include calculating the iron losses in magnetic components and the design of inductors used in power electronics circuits.
Toshihisa Shimizu (Fellow) received the B.E., M.E. and Dr. Eng. de-

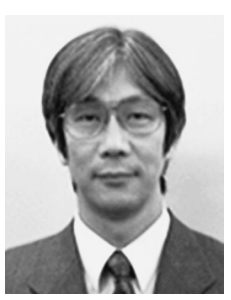
grees in electrical engineering from Tokyo Metropolitan University in 1978, 1980, and 1991, respectively. He joined Fuji Electric Corporate Research and Development, Ltd., in 1980. Since 1993, he has been with the Department of Electrical Engineering, Tokyo Metropolitan University, where he is currently a Full Professor. His research interests include power converters, high-frequency inverters, photovoltaic power generations, EMI in power converters, high power density converter design, and passive components for power converters.

Kinji Kanagawa (Member) received his B.S. from Keio University,

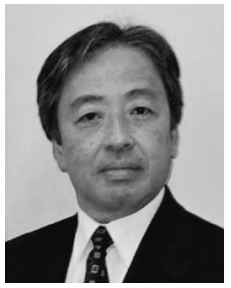
Yokohama, Japan in 1978. He joined Mitsubishi Materials Corporation in 1992. 